\title{
Adrien Lucca
}

Researcher, Fine Art, Jan van Eyck Academie, Maastricht, Netherlands Comité de rédaction de l'Isfa, International Society of the Friends of Aetherometry

\section{(2012)}

\section{"Wilhelm Reich. Biographie critique."}

Un document produit en version numérique par Jean-Marie Tremblay, bénévole, professeur de sociologie retraité du Cégep de Chicoutimi

Courriel: jean-marie_tremblay@uqac.ca

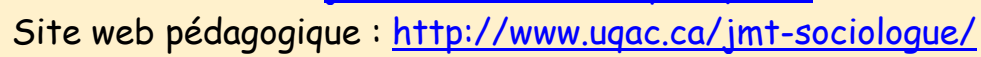

Dans le cadre de: "Les classiques des sciences sociales" Une bibliothèque numérique fondée et dirigée par Jean-Marie Tremblay, professeur de sociologie au Cégep de Chicoutimi

Site web: http://classiques.ugac.cal

Une collection développée en collaboration avec la Bibliothèque Paul-Émile-Boulet de l'Université du Québec à Chicoutimi Site web: $\underline{h t+p: / / b i b l i o t h e q u e . u q a c . c a l}$ 


\section{Politique d'utilisation de la bibliothèque des Classiques}

Toute reproduction et rediffusion de nos fichiers est interdite, même avec la mention de leur provenance, sans l'autorisation formelle, écrite, du fondateur des Classiques des sciences sociales, Jean-Marie Tremblay, sociologue.

Les fichiers des Classiques des sciences sociales ne peuvent sans autorisation formelle:

- être hébergés (en fichier ou page web, en totalité ou en partie) sur un serveur autre que celui des Classiques.

- servir de base de travail à un autre fichier modifié ensuite par tout autre moyen (couleur, police, mise en page, extraits, support, etc...),

Les fichiers (.html, .doc, .pdf, .rtf, .jpg, .gif) disponibles sur le site Les Classiques des sciences sociales sont la propriété des Classiques des sciences sociales, un organisme à but non lucratif composé exclusivement de bénévoles.

Ils sont disponibles pour une utilisation intellectuelle et personnelle et, en aucun cas, commerciale. Toute utilisation à des fins commerciales des fichiers sur ce site est strictement interdite et toute rediffusion est également strictement interdite.

L'accès à notre travail est libre et gratuit à tous les utilisateurs. C'est notre mission.

Jean-Marie Tremblay, sociologue

Fondateur et Président-directeur général, LES CLASSIQUES DES SCIENCES SOCIALES. 
Cette édition électronique a été réalisée par Jean-Marie Tremblay, bénévole, professeur de sociologie au Cégep de Chicoutimi à partir de :

Adrien Lucca, Comité de rédaction de l'ISFA

"Wilhelm Reich. Biographie critique".

Concord, Ontario, Canada : Texte inédit produit pour Les Classiques des sciences sociales à la demande du Dr Paulo Correa, Director R\&D, Aurora Biophysics Research Institute, 8 février 2012.

[Autorisation formelle accordée par l'auteur le 8 février 2012 de diffuser ce texte dans Les Classiques des sciences sociales.]

Courriel : drpcorrea@abrisci.com

Polices de caractères utilisée : Comic Sans, 12 points.

Édition électronique réalisée avec le traitement de textes Microsoft Word 2008 pour Macintosh.

Mise en page sur papier format : LETTRE US, 8.5" $\times 11^{\prime \prime}$.

Édition numérique réalisée le 8 février 2012 à Chicoutimi, Ville de Saguenay, Québec. 
Adrien Lucca, comité de rédaction de I'ISFA

International Society of the Friends of Aetherometry

\section{Wilhelm Reich. \\ Biographie critique.}

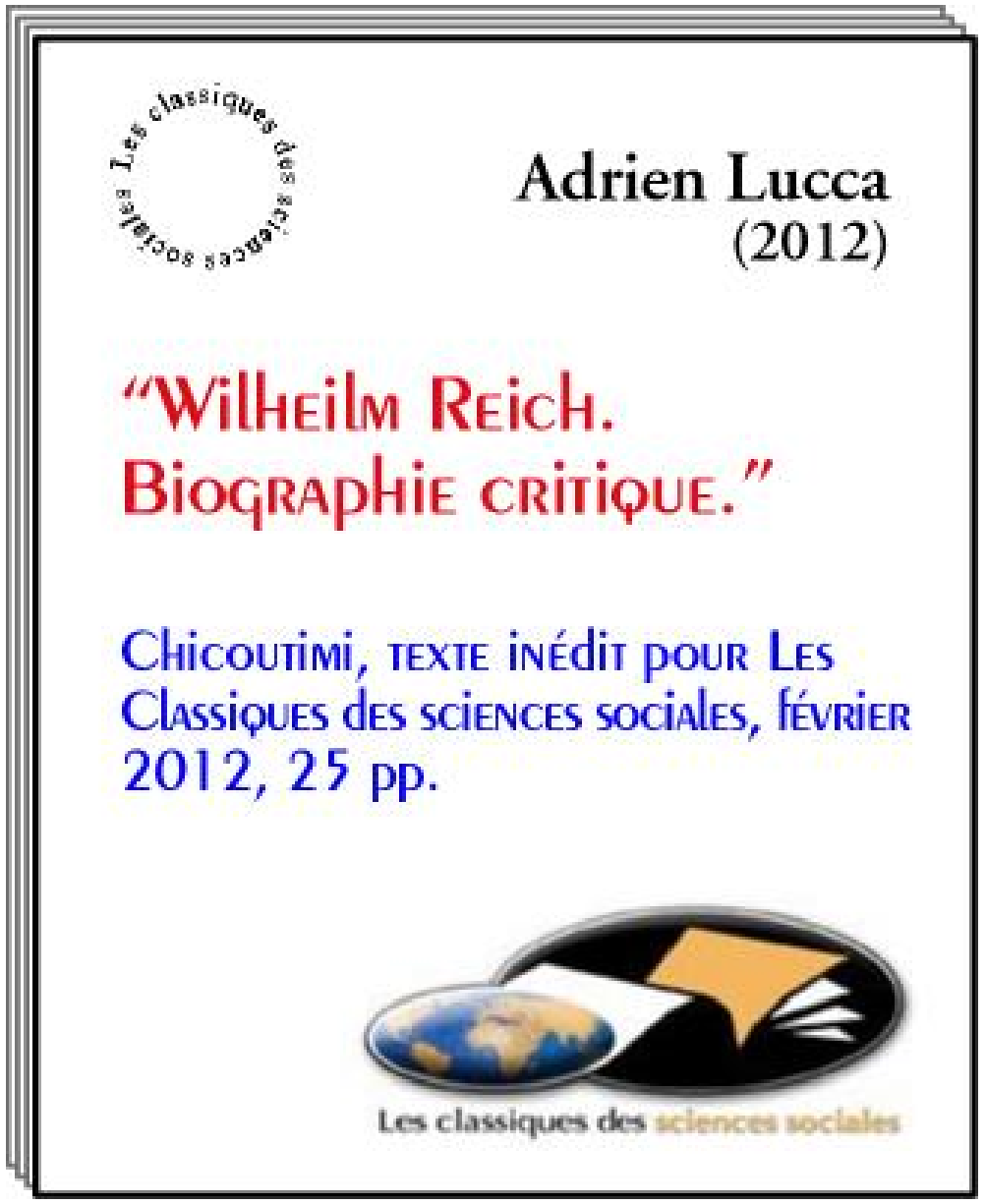

Concord, Ontario, Canada: Texte inédit produit pour Les Classiques des sciences sociales à la demande du Dr Paulo Correa, Director R\&D, Aurora Biophysics Research Institute, 8 février 2012. 


\section{Table des matières}

Introduction. Wilhelm Reich

VIE EN EUROPE

1. La Période de Formation, 1897-1922

2. La Période Psychanalytique, 1922-1930

3. La Période Freudomarxiste, 1930-1936

4. La fuite du fascisme et la Période des Bions sur les origines de la vie, 1933-1939

\section{VIE EN AMÉRIQUE}

5. La Période de la Découverte de l'Énergie de l'Orgone, 19391946

6. L'Invention du "Moteur d'Orgone" et la "Clé du Cancer", 19461950

7. La Découverte de l'énergie de la DOR (Deadly ORgone), 19501956

8. La Période de Persécution Judiciaire, Prison et Mort, 1954-1957

9. L'Influence des Travaux de Reich, 1957-2011 


\section{Adrien Lucca * , Comité de rédaction de I'ISFA ** \\ "Wilhelm Reich. Biographie critique."}

Concord, Ontario, Canada : Texte inédit produit pour Les Classiques des sciences sociales à la demande du Dr Paulo Correa, Director R\&D, Aurora Biophysics Research Institute, 8 février 2012.

\section{Wilhelm Reich}

\section{Retour à la table des matières}

Wilhelm Reich, Juif Autrichien naturalisé Américain - né le 24 mars 1897 à Dobrzanica (Autriche-Hongrie, aujourd'hui en Ukraine), mort le 3 novembre 1957 au pénitencier fédéral de Lewisburg, Pennsylvanie, États-Unis d'Amérique - était médecin, psychiatre, psychanalyste, philosophe, sociologue, biophysicien, inventeur et antifasciste. Après la première guerre mondiale, il fut surtout célèbre en tant que principal théoricien du Freudo-marxisme, pour son analyse profonde et originale de la plaie du fascisme ainsi que pour son opposition contre Freud sur la question de l'instinct de mort. Plus tard, après la seconde guerre mondiale, il fut connu comme le découvreur d'un type nouveau d'énergie, sans masse ni poids, partout présente dans l'atmosphère et qu'il estimait si fondamentale à la genèse de la vie et aux fonctions biologiques qu'il l'identifia à la Libido freudienne et la nomma "Orgone".

\footnotetext{
Researcher, Fine Art, Jan van Eyck Academie, Maastricht, Netherlands.

** International Society of the Friends of Aetherometry.
} 
Récemment encore, aucun scientifique n'avait tenté une vérification systématique des découvertes de Reich dans les domaines de la physique, biologie, oncologie, biochimie et biophysique. Dès les années 2000, les travaux du Dr. Paulo Correa, d'Alexandra Correa et de leurs collaborateurs à l'Aurora Biophysics Research Institute ont reproduit la quasi-totalité des travaux de Reich, confirmant beaucoup de ses découvertes - mais mettant également en lumière et expliquant nombre de ses erreurs dans tous les domaines de recherche cités ci-dessus.

\section{VIE EN EUROPE}

\section{La Période de Formation, 1897-1922}

\section{Retour à la table des matières}

Fils de Leon Reich, un fermier aisé, et de Cecilia Roniger, Juifs assimilés, le jeune Reich eut l'occasion de se faire autodidacte. À l'âge de 14 ans, il joua un rôle important dans le suicide de sa mère, à la suite des liaisons de celle-ci et des assauts qu'elle subit de son mari. À 18 ans, après la mort de son père en 1914, Reich fut appelé dans l'armée Austro-hongroise alors en pleine guerre. Suite à la chute de Bukovina, la ferme et tout l'héritage familial furent perdus à jamais, les jetant son frère et lui dans la pauvreté. Dans son autobiographie Passion of Youth 1 , il écrit: "Je ne revis jamais ni ma terre natale, ni mes biens. De ma vie passée, il ne m'est rien resté." Le jeune Reich participa alors à l'offensive Autrichienne et Allemande d'Izonso contre les Italiens. Les années de guerre lui laissèrent un sens profond des injustices commises par les Autrichiens contre les populations civiles, il décrivit la guerre comme une psychose collective. Commencera alors sa critique des principes du Pouvoir et de l'autorité ainsi que sa recherche des racines du militarisme. Après la guerre, Reich reprit ses études médi-

1 Reich W (1937) "Passion of youth - an autobiography, 1897-1922", Farrar, Straus \& Giroux, NY, 1988 Éd. 
cales dans l'Ecole de Médecine de Vienne, qu'il achèvera avec distinction à l'âge de 25 ans.

La pensée d'Henri Bergson influença Reich profondément. Bergson énonça une philosophie vitaliste située à la fois au-delà du mécanisme et du finalisme, fondée sur le concept central d'une force créatrice régissant la vie: "l'élan vital". Reich désirait apporter au bergsonisme les fondements biologiques et biophysiques qui lui manquaient en considérant cette force d'un point de vue scientifique, comme la manifestation d'une "énergie vitale". On peut même voir ici le plan le plus général du programme de recherche qu'il s'assignera durant toute sa vie. Pendant la période Américaine de Reich, l'élan vital deviendra l'expression de l'autorégulation de l'Orgone dans les systèmes biologiques.

\section{La Période Psychanalytique, 1922-1930}

\section{Retour à la table des matières}

Entre 1922 et 1924, Reich travaillera dans la Faculté de Médecine Interne de l'Hôpital de l'Université de Vienne. Il y étudiera la neuropsychiatrie sous l'égide du Professeur Wagner-Jauregg (qui obtint le Prix Nobel en 1927). En 1920, Reich deviendra aussi le plus jeune membre de l'Association Psychanalytique de Vienne. Il sera alors l'élève préféré de Sigmund Freud. Entre 1924 et 1930, il sera chargé avec Sandor Ferenczi de la codirection de l'important Séminaire de Technique Psychanalytique - il est alors considéré comme l'héritier de Freud. En 1928, il devint vice-directeur de la Polyclinique Polyanalytique de Vienne ouverte par Freud. Pendant cette période Viennoise, il commencera sa recherche sur les causes de la névrose et sur de nouvelles méthodes thérapeutiques.

Très tôt, Reich voulut fournir une base scientifique à l'hypothèse freudienne de la "Libido" (l'énergie sexuelle de l'inconscient), dont l'économie serait régie par le "Principe de Plaisir", mais soumise à la répression sexuelle et sociale du "Principe de Réalité". Cependant, Freud s'était distancié de cette hypothèse dès 1920: dans Au-delà du Principe de Plaisir il proposait une dualité fondamentale de 
l'inconscient (Eros/Thanatos) - où la Libido voudrait autant la mort ("Une compulsion à répéter le non-plaisir"), que la vie.

Reich s'opposa à ce tour de force de la théorie et du diagnostic psychanalytiques. En 1924, il présentera à Freud sa solution dans le manuscrit La fonction de l'Orgasme (Die funktion des Orgasmus), que Freud rejeta. Reich le publiera néanmoins en 1927. Il croyait alors que sa découverte de la fonction énergétique de l'orgasme au cœur du désir inconscient démontrait que l'économie libidinale ("sexuelle") possédait désormais une base physiologique, clinique et psychiatrique. Il n'y avait dans l'inconscient que le Principe de Plaisir : "L'instinct sexuel n'est autre que la mémoire motrice d'un plaisir passé" 2 . Reich s'opposa fermement au nouveau Freud lorsque ce dernier affirma que l'inconscient était également régi par l'instinct de mort, qui de plus était toujours victorieux. Au même moment, Freud proclamait la grandeur d'hommes d'États tels que Mussolini ou le socialiste-chrétien Engelbert Dollfuss, "héros de la civilisation", seuls capables de maitriser Thanatos... Dans une copie du bouquin Pourquoi la Guerre (avec Einstein comme coauteur), une dédicace à Mussolini signée par Freud proclame : "D'un vieil homme qui salue dans le Duce un héros de la culture".

Il n'y a pas d'instinct de mort à l'œuvre dans le désir! - s'écrira Reich, mais des processus socio-historiques de répression du désir qui créent un réservoir d'énergie non déchargée, retenue et immobilisée dans le corps au moyen d'un état de tension musculaire chronique. Débute alors sa théorie de la cuirasse caractérielle, publiée dans L'Analyse du Caractère ${ }^{3}$, dont la première partie - traitant du caractère masochiste - fut publiée par le journal de l'association psychanalytique en 1932, ce non sans l'interférence de Freud qui voulait y joindre sa propre critique du travail de Reich.

Selon Reich, le caractère névrotique est maintenu par la répression sexuelle qui dévie l'énergie sexuelle pour l'investir dans la cuirasse, la

2 Reich W (1927) "The function of the orgasm", Simon \& Schuster, NY, 1973 ed., p. 51. La traduction est de nous. "La fonction de l'orgasme", L'Archee, Paris, 1952.

3 Reich W (1933, 1945) "Character-analysis", Farrar, Straus \& Cudahy, NY, 1949 edition. Traduit en Fr. par Kamnitzer sous le titre "L'analyse caractérielle", Payot SH, 1973. 
névrose étant fondée par une perturbation de la libido du système nerveux autonome (ou végétatif), centrée sur l'impuissance orgastique. Reich nommera cette perturbation "sympathicotonie chronique", c'està-dire la condition acquise de contraction systématique d'une branche du système nerveux végétatif formé par tous les nerfs orthosympathiques. Il était alors clair que la sympathicotonie chronique qui ancrait la névrose était une dys-économie inconsciente de la Libido, tandis que la cause directe du contenu des névroses serait d'ordre politique (soumission au Patriarcat, aux chefs, à la moralité, à la proscription de la liberté et de l'avortement, etc.) et d'ordre socio-économique (malnutrition, exploitation, problèmes de logement, dépendance économique de la femme, etc.) La répression sexuelle n'était autre qu'une internalisation de la répression sociale sous toutes ses formes, que la prolongation inconsciente du Pouvoir patriarcal.

\section{La Période Freudomarxiste, 1930-1936}

\section{Retour à la table des matières}

Les critiques que Reich adressa à Freud, soit à propos de la nature de la libido soit au sujet des origines sociales de la névrose, le menèrent directement à l'étude des processus historiques tracés par les institutions sociales, y inclus la relation entre la culture et le système sauvage ou tribal de la famille ainsi que l'émergence des institutions politiques, militaires et économiques au fil de la Préhistoire et de l'Histoire.

Sa théorie sexuelle dénonçait en effet ce qu'Alfred Jarry avait ridiculisé sous l'appellation de SurMâle. La répression sexuelle servait une société dominée par les patriarches, surtout blancs et Européens qui détenaient le pouvoir sous toutes ses formes: pouvoir des richesses, pouvoir de l'État, pouvoir militaire et pouvoir dans la famille. C'était juste avant la prise du Pouvoir par les Nazis, alors que le Vieux Monde Chrétien patriarcal existait encore. Reich saisit alors la jonction - qu'il estimait parfaite - entre ses théories analytiques du désir et de la personnalité et la théorie historique et économique des sociétés avancée par Karl Marx et Friedrich Engels: ce fut sa période freu- 
domarxiste. Son concept restrictif de l'économie sexuelle pouvait bien définir un dynamisme propre à la sphère des structures idéologiques dans le schéma marxiste. C'aurait été ce que Marx et Engels cherchaient dans L'Idéologie Allemande mais qu'ils n'avaient pas su analyser scientifiquement. De ce point de vue, le marxisme devrait être remanié, car "la vraie dialectique" contenait autant de surdéterminations politico-économiques de la subjectivité et de ses idéologies du possible qu'elle contenait de conditionnalismes d'ordre libidinal, désirant, subjectif, qui relèveraient tous de la pathologie sexuelle et repousseraient le progrès des surdéterminations politico-économiques, liées au développement historique de l'infrastructure sociale.

En somme, dans la pensée freudomarxiste de Reich de cette époque, la superstructure idéologique interagissait avec l'infrastructure politico-économique. L'action des deux structures était mutuelle. La révolution sociale devrait alors s'accomplir par une révolution de la vie sexuelle des masses, par un travail d'hygiène mentale, sexuelle, touchant le corps et la cuirasse. Dans le but d'accomplir ce programme, Reich - qui était membre du Parti Communiste Autrichien dès 1928 partit pour Berlin, adhéra en 1930 au Parti Communiste Allemand (le KPD) et organisa le mouvement SexPol, créant des cliniques dont les objectifs étaient de soigner les problèmes et désordres sexuels des travailleurs et des pauvres, d'offrir des séances analytiques gratuites et d'enseigner la sexologie. Le mouvement organisa et soutint également un centre public de recherche et de discussion sur la vie sexuelle des gens, surtout des jeunes, et publia un journal, le Zeitschrift für Politische Psychologie und Sexualökonomie. Le fonctionnement de ces cliniques échappait aux contrôles respectifs de L'Association Psychanalytique et du KPD.

Suivant sa conclusion que l'analyse devrait embrasser la théorie marxiste de la société, Reich adopta la théorie du Matriarcat de Bachofen et Engels, attribuant toutes les oppressions sociales au $\mathrm{Pa}-$ triarcat dans son livre L'Irruption de la Morale Sexuelle ${ }^{4}$. Les origines de l'État, de la propriété privée et du Capital, du militarisme et de

4 Reich W (1932) "Der Einbruch der Sexualmoral", Verlag für Sexualpolitik, Berlin. Traduit en Fr. par Kamnitzer sous le titre "L'iruption de la morale sexuelle", Payot SH, 1972. 
la guerre des classes, pouvaient toutes êtres ramenées aux changements préhistoriques de la famille dite matriarcale des sauvages à la famille patriarcale. Au SurMâle dominant, Reich opposa le "caractère génital" de l'homme et la femme sachant aimer et jouir dans l'épanouissement orgastique et la créativité intellectuelle, artistique et scientifique.

Tout ceci déplut fort aux stalinistes et au KPD dans l'Allemagne pré-Nazie. La pensée de Reich était en fait très proche du "marxisme libertaire", des positions anarcho-syndicalistes et anarchocommunistes alors courantes en Espagne, juste avant la guerre civile. Sous le pseudonyme E. Parell, il publia son remaniement du marxisme en concluant: "La SexPol concorde essentiellement avec les propos anarchistes, mais sans pour autant abandonner les conceptions marxistes de la structure économique ou le rôle du parti [communiste]. (...) On peut même dire que la SexPol est l'union de l'anarchisme et du marxisme sur une base marxiste." 5

Le Freudo-marxisme de Reich fut un effort pour sauver le marxisme alors que la "pratique" des pires interprétations de celui-ci (le léninisme, le stalinisme, etc) n'était pas encore assouvie, et alors même que son spectacle d'opposition aux fascismes faisait semblant d'arrêter leur montée. Il est cependant clair aujourd'hui que le Freudomarxisme fut une grande erreur, tantôt analytique, tantôt politique, sociologique et historique - car l'économie libidinale n'est pas le côté subjectif-irrationnel de l'économie politique, sa surdétermination, mais le désir même, produisant et l'économique et le politique. En d'autres termes, s'il y en a oppression, c'est que ceux qui sont assujettis en veulent bien davantage : le désir étant de surcroit désir de sa propre répression. Suivant Gilles Deleuze et Félix Guattari 67 , ce qui manquait

5 Parnell E (aka Reich W) (1936) "Reply to certain of the anarchist comrades' objections", dans "The International Sex-Pol Discussion".

6 Deleuze G \& Guattari F (1973) "L'anti-Oedipe - capitalisme et schizophrénie", Les Éditions de Minuit, Paris.

7 Correa PN \& Correa AN (1979) "A monist view of the relation between libidinal and political economies and the problem of fascism", Akronos Publishing, Concord, Canada, ABRI monograph AS2-05, repris dans Philos of Sci \& Polit of Thought, Vol. 1, 5:1-11 (May 2002). 
à Reich à l'époque, c'était de faire la découverte qu'il n'y a jamais eu qu'une seule économie: que celle-ci soit saisie comme subjectivité créatrice (inventive, législative, souveraine), comme "économie libidinale" - ou comme une machine géante avec des institutions de Pouvoir (toute une économie politique) qui projettent les formes de la subjectivité soumise. En termes deleuzo-guattariens, il lui manquait la catégorie de "la production désirante" pour que sa théorie de la libido puisse échapper au freudisme et au marxisme.

De plus - on le sait aujourd'hui grâce aux travaux de Lévi-Strauss, de Pierres Clastres et de bien d'autres - un Patriarcat a beau avoir eut lieu, il n'y a jamais eu ni Matriarcat ni aucun état sauvage sans pratiques guerrières. Nous savons aussi que l'État n'est pas né d'un détraquage de la dette finie du système de Potlatch comme le croyaient Reich et Malinowski - ni d'une période de guerre des classes originelle comme l'avaient cru Engels et Lénine. L'État militariste, et même l'État despotique, peuvent bien être patriarcaux sans pour autant avoir eu une origine économique. Le marxisme s'avère en fait incapable de comprendre la société sauvage, la naissance de l'État et la formation d'États militaires.

\section{La fuite du fascisme et la Période des Bions sur les origines de la vie, 1933-1939}

\section{Retour à la table des matières}

Les idées et les travaux de Reich - y compris sa pratique médicale menaçaient Freud, son Association Psychanalytique autant que le KPD et son maitre Staline. Après la montée d'Hitler au pouvoir en janvier 1933, Reich fuit pour Copenhague mais retourne en Autriche lorsque Freud décide que l'Association Psychanalytique ne publiera pas son livre L'Analyse du Caractère. Commencèrent alors les persécutions jésuitiques dont il fut victime de la part des militants communistes et des analystes freudiens. Reich se réfugie au Danemark, où il est expulsé du Parti Communiste Danois - auquel il n'a jamais appartenu! Pour rester au Danemark, Reich avait besoin du soutien officiel de Freud: celui-ci le refusa et Reich fut aussi expulsé du Danemark. Il fut éga- 
lement exclu du KPD en 1933 à la suite de la publication de son livre La Sexualité des Jeunes. En mai 1934, il fut expulsé de Suède et en août, du Congrès de l'Association Psychanalytique à Lucerne. C'est à la suite des efforts malveillants de Paul Federn et d'Ernest Jones en vue de l'exclure de l'Association Psychanalytique que Reich, en ayant assez, renonce à y appartenir. Il s'exile alors en Norvège, à Oslo, en octobre 1934.

C'est alors que ses livres sont brulés dans les universités par les Nazis - qui mettent son nom dans la liste des auteurs Juifs et antifascistes censurés - que Reich se replonge dans son travail de recherche. Il explore les fonctions électriques de la sexualité et de l'angoisse 8 et découvre que sa formule de l'orgasme - alternance entre expansion et contraction - définit également la pulsation de toutes les cellules et caractérise ainsi tous les systèmes vivants. Ces études lui font penser que la névrose, ainsi que tous les désordres sympathicotoniques, impliquent des perturbations électriques et chimiques.

Reich estime aussi que la vie elle-même est née de la combinaison de la matière et de l'énergie électrique et que les conditions de laboratoire nécessaires à sa reproduction expérimentale devraient joindre processus chimiques (tel l'absorption d'eau induite par le potassium) et mécaniques (tel l'effet de la haute chaleur). Sa théorie abiotique de la biogénèse ne se situait pas en dehors du cadre scientifique de l'époque. Les théories d'Oparin étaient courantes dès les années 1920, et Stanley Miller démontra en 1953 qu'il était possible de produire presque tous les acides aminés qui entrent dans la composition des protéines en faisant passer des décharges électriques issues d'une bobine de Tesla à travers une mixture d'ammoniaque, de méthane, d'hydrogène et de vapeur d'eau.

Après le traitement chimique et la mise à incandescence de nombreuses substances (charbon, cristaux de fer, sable de mer), Reich découvre au microscope - en dépit des techniques d'hyper-stérilisation employées - qu'il obtient toujours des vésicules qui ressemblent et aux bactéries et aux vésicules que l'on retrouve dans la plupart des cellules 
eucaryotes (aujourd'hui appelées "organelles" dans leur diversité). Il décompose une grande variété de cellules eucaryotes et observe que certaines vésicules résistent à la stérilisation. Il en conclut que la matière et les cellules vivantes se décomposent en général en deux types de vésicules - qu'il nomme pour l'un "bions PA" et pour l'autre "bacillus$T^{\prime \prime}$. Les bions PA ressemblent aux cocci (chaque coccus mesurant entre 0,7 et $4 \mu \mathrm{m}$ de diamètre), en particulier les micrococci et sarcinae qui s'organisent en paquets. Selon Reich, ils peuvent former des agrégats du type staphylo ou de type géométrique comme des sarcinae. Les bacillus- $T$, eux, sont des objets submicroscopiques (ou nanoscopiques) mesurant environ $250 \mathrm{~nm}$. Pour les observer, Reich s'est servi de microscopes montés de puissants objectifs achromatiques et d'oculaires compensatoires, et employa la technique de "l'ultra-condensateur de champ noir".

En conséquence du fait qu'il obtint ces bions en dépit de l'usage de techniques de stérilisation dépassant largement les critères définis par Louis Pasteur, il conclut que ses expériences devraient démontrer la possibilité d'une création de micro-organismes (une forme de "vivant procaryotique") à mi-chemin entre la matière et le vivant - vivant qu'il conçut presque toujours comme un "vivant eucaryotique". Ces expériences constitueraient donc selon lui la preuve de l'abiogenèse des procaryotes.

Puisque les bions PA s'assemblent en paquets, Reich pense aussi que ces paquets peuvent former des cellules eucaryotes, une ou plusieurs vésicules de l'agglomérat se transformant en noyau cellulaire. Ce serait là la clé de l'abiogenèse des eucaryotes. On peut aujourd' hui apprécier à quel point sa théorie de la genèse des eucaryotes est proche de la théorie endosymbiotique de Lynn Margulis publiée en 1976, quarante années plus tard.

Reich commit pourtant ici deux de ses pires erreurs. Il ne suffit pas d'obtenir, en dépit de l'hyper-stérilisation, des micro-organismes de sources variées pour conclure à l'abiogenèse des procaryotes. Comme il l'avoue presque lui-même, il suffirait que des spores thermorésistantes soient présentes dans les matériaux-sources, ou que des organismes hyper-thermophiles existent. Sa seconde erreur découla d'une confusion entre les paquets de cocci et les amoebae - qui est à l'origi- 
ne du nom "PA" ("Packet Amoebae") des bions-PA. Cette erreur sera pleine de conséquences pour sa théorie du cancer, car les paquets de cocci (ou bions-PA), ne sont pas des organismes protozoaires, pas plus qu'ils ne se transforment en ceux-ci.

\section{VIE EN AMÉRIQUE}

\section{La Période de la Découverte de l'Énergie de l'Orgone, 1939-1946}

\section{Retour à la table des matières}

La montée de l'Europe fasciste contraignit Reich à fuir pour I'Amérique. À la suite de sa rencontre avec Malinowski en Angleterre en 1939 - qui obtiendra pour Reich une position d'enseignant à la New School for Social Research, New York - Reich arriva aux États-Unis en 1940. Mais après l'attaque de Pearl Harbor, il fut arrêté pour suspicion d'être un Nazi par ordre de J. Edgar Hoover lui-même ${ }^{9}$, ce qui lui valut trois semaines de prison à Ellis Island.

En 1942, il achète une grande propriété près de Rangeley (Maine) qu'il baptisera Orgonon, d'après le nom de sa découverte: l'Orgone. Il y installa l'Orgone Institute, son propre laboratoire ainsi que des laboratoires d'instruction accueillant des cours publics. Jusqu'en 1954 y eurent lieu des conférences abordant ses idées, découvertes et inventions ainsi que l'évolution de ses recherches. L'institut publiera un journal, l'Orgone Energy Bulletin (dont tous les numéros, ainsi que ceux des autres revues éditées par Reich, sont disponibles en microfilms à la bibliothèque Sainte Geneviève de Paris). Orgonon deviendra le centre des activités de Reich, de ses assistants et élèves. C'est actuellement le lieu où se situe le Wilhelm Reich Museum. Quand Reich se rendra compte du fait que la réception de ses découvertes sur l'Orgone ne sera pas objective, que ses travaux seront rejetés puis déformés par les médias de façon conspiratrice et ce dans le but qu'ils soient

9 FOIA USA, "FBI file on Dr. Wilhelm Reich", Bufile:100-14601, Section 1. 
supprimés, il conclura que seule l'éducation des enfants dès le plus jeune âge pourra ouvrir leurs corps et leurs esprits aux réalités de l'énergie d'Orgone. Il créa alors l'Orgonomic Infant Research Center, dédié à l'éducation et à l'étude de l'enfant depuis le stade prénatal jusqu'à l'adolescence. Il y établit aussi l'Orgone Energy Clinic, dédiée au traitement des maladies sympathicotoniques à l'aide de ses méthodes bioénergétiques.

La découverte de l'Orgone se fut en deux temps bien distincts. En 1939, à la suite d'une erreur de sa femme Ilse Ollendorff dans une préparation de Bions, du sable d'océan fut mis à incandescence à la place du charbon. Des paquets réguliers de cocci bleuâtres et très brillants dans le champ noir microscopique en résultèrent (il les nomma "bions SAPA", pour: Sand Packet Amoeba). Reich commit alors l'erreur d'attribuer la brillance bleuâtre à une émission spontanée des bions SAPA. Après qu'il eût laissé une paire de gants sur les échantillons SAPA et que ceux-ci eurent acquis une forte charge électrostatique capable de charger un électroscope, Reich conclut - encore une fois de manière erronée - qu'il avait découvert une énergie biologique pouvant charger électriquement les matériaux diélectriques. Et puisqu'il concevait les bions SAPA comme des micro-organismes créés par "génération spontanée", il ajouta encore que tout ceci était la preuve que cette énergie électrique était l'énergie créatrice de la vie. Dans les tentatives faites par la suite en vue d'isoler la radiation bleuâtre, Reich remarqua cependant que celle-ci était partout, hors des échantillons. Afin de la délimiter il construisit pour la première fois des cages de Faraday, dans lesquelles il observa les mêmes luminescences ou scintillations bleuâtres 10 .

Récemment, les Correas ont reproduit les expériences de Reich sur les bions et ont identifié les bions SAPA à des sarcinae océaniques, capables de produire des spores qui semblent être hyperthermophiles ${ }^{11}$. Ils ont également démontré que la brillance bleuâtre des bions SAPA dépendait de l'excitation induite par une lampe au

10 Sharaf M (1983) "Fury on earth - a biography of Wilhelm Reich", St. Martin's Press, NY, p. 277.

11 Correa PN \& Correa AN (2010) "The orgonomic theory of cancer", J Biophys Hematol Oncol, 1(3):1. 
mercure et qu'elle n'est donc pas spontanée ${ }^{12}$. Les scintillations observées dans un accumulateur ne sont de toute façon pas du même ordre que les réfractions intenses des bions SAPA.

Sa seconde découverte de l'Orgone ne fut en revanche pas jonchée d'erreurs, mais marquée de véritables percées. Reich avait observé qu'une boite métallique du genre "cage de Faraday" était spontanément capable d'élever la température juste au dessus d'elle et à l'intérieur, et que s'il couvrait la boite d'une couche isolante la différence de température augmentait. Cette différence était plus importante lors des journées anticycloniques que lors des journées cycloniques - et maximale s'il exposait les boites directement à la lumière solaire. De plus, un électroscope placé à l'intérieur d'une boite perdait sa charge beaucoup plus lentement qu'un électroscope témoin placé au dehors. Il en conclut que la radiation solaire était la source d'une énergie qui: (1) luisait ou scintillait par elle-même, (2) était capable de charger électriquement l'électroscope et les substances isolantes, et que (3) cette énergie se transformait en chaleur lorsqu'elle était barrée dans sa propagation ou son mouvement. Puisque celle-ci traversait ou se propageait à travers tous les milieux, qu'ils soient conducteurs ou isolants, il conclut que cette énergie était sans masse ou sans inertie: "massfree energy".

Encouragé, Reich décide alors d'obtenir confirmation de sa découverte auprès d'Albert Einstein. Après une rencontre qui dura 5 heures, Einstein voulut répéter pendant une semaine l'observation de la différence de température au dessus de la boite de Faraday. Cette expérience, connue sous le nom d' Expérience de Reich-Einstein, vit ses résultats confirmés par les Correas et indépendamment par Eugene Mallove 13141516 . Einstein confirma bien la différence de température

12 Correa PN \& Correa AN (2010) "The PA \& SAPA bion experiments and protoprokaryotic biopoiesis", J Biophys Hematol Oncol, 1(2):1.

13 Correa PN \& Correa AN (2001) "The reproducible thermal anomaly of the ReichEinstein experiment under limit conditions", Infinite Energy, 37:12. Repris dans $J$ Aetherom Res, 2(6):1.

14 Correa PN \& Correa AN $(1998,2001)$ "The thermal anomaly in ORACs and the Reich-Einstein experiment: implications for blackbody theory", Akronos Publishing, Concord, Canada, ABRI monograph AS2-05. 
comme étant un résultat positif - mais sans aucun esprit critique, il accepta une explication insuffisante pour la justifier 17 , explication suggérée par le physicien Polonais Leopold Infeld, alors son assistant. Reich riposta avec de nouveaux résultats, mais Einstein resta toujours muet à leur sujet. Les documents de cette affaire furent publiés en 1953 par l'Orgone Institute Press dans un recueil intitulé The Einstein Affair.

Cette énergie que Reich associa (à cause des SAPA) à l'élan vital de Bergson et qu'il baptisa "énergie d'Orgone", il l'avait d'abord considérée comme étant électrique. La seule différence envers d'autres formes d'énergie étant l'absence d'inertie de ses particules. Mais au fur et à mesure de ses recherches sur la nature de l'Orgone, Reich s'est lui-même convaincu qu'elle n'était ni électrique ni électromagnétique. Il commença alors un ambitieux programme d'enquête sur la physique de l'Orgone 18, qui fut en partie une entreprise de redécouverte de l'électricité statique, de l' "électricité animale" de Luigi Galvani - ou de I' "électricité longitudinale" de Nikola Tesla. En employant des détecteurs de particules - des tubes Geiger-Müller insérés dans des accumulateurs d'Orgone spéciaux - Reich crut découvrir une pulsation de fond ("background rate") qu'il attribua à l'Orgone. Il pensa alors que cette pulsation était capable de maintenir la charge - qu'il estimait être orgonotique, et non électrique -, des électroscopes chargés par transferts d'Orgone au moyen d'un bâton électrostatique. Il fit la commande de tubes de type diode (qu'il nomme VACOR) ayant un vide de l'ordre du microtorr, et découvrit qu'il pouvait leur faire produire

15 Mallove E (2001) "Breaking Through: A Bombshell in Science", Infinite Energy, 37:6. Repris dans $J$ Aetherom Res, 2(6):25.

16 Comité de Rédaction de la ISFA (International Society of the Friends of Aetherometry) (2006) "The Reich-Einstein experiment".

17 Correa PN \& Correa AN (2001) "The reproducible thermal anomaly of the Reich-Einstein experiment under limit conditions", Infinite Energy, 37:12. Repris dans J Aetherom Res, 2(6):1.

18 Reich W (1951) "The Orgonomic anti-nuclear radiation project (ORANUR) report", Orgone Energy Bull, 3(4):185. 
des taux de pulsation très grands pour l'époque (il nomme le taux de pulsation de $25 \mathrm{kps}$ le REICH-ORG ${ }^{19}$ ).

Il conclut de tout ce qui précède que:

1. I'Orgone est composé de charges sans-masse, électrostatiques, ayant un potentiel très élevé.

2. les ondes émises pas une bobine d'induction ne sont pas électromagnétiques, mais des ondes de Tesla avec leurs charges sans-masse et électrostatiques.

3. I'Orgone agit transversalement à la direction des champs électriques 2021 [19-20], elle est donc selon lui "unipolaire".

D'après les travaux des Correas et de leurs collaborateurs, notamment le Dr. Eugene Mallove et la Dr. Malgosia Askanas, Reich a commis des erreurs très importantes lors de cette période. La pire d'entre elles fut de ne pas contrôler la réponse électrique des électroscopes chargés par des bâtons électrostatiques. S'il l'avait fait, il aurait remarqué que la méthode qu'il avait choisie transférait toujours, non pas de l'Orgone, mais de l'électricité négative ordinaire. Cela aurait eu de lourdes conséquences sur son programme. Il aurait alors observé comme l'ont fait les Correas - qu'un électroscope chargé positivement, placé dans le même accumulateur d'Orgone, ne se décharge ni plus ni moins vite qu'un autre chargé négativement, ce qui lui aurait prouvé que l'énergie qui, par son accumulation dans les boites, ralentit la décharge spontanée de l'électroscope n'est ni électrique ni électrostatique, et qu'elle ne charge pas l'électroscope comme il le prétendait. En effet, d'après les travaux expérimentaux des Correas, c'est bien

19 Ibidem, p. 266.

20 Reich W, Remarques 4-8 Août 1940, dans "American Odyssey - letters and journals, 1940-1947", Farrar, Straus \& Giroux, NY, 1999, p. 32.

21 Reich W, Lettre à Einstein, 20 Février 1941, dans "American Odyssey - letters and journals, 1940-1947", 1999, p. 74. 
l'accumulation de chaleur latente 2223242526 qui est responsable à la fois du ralentissement de la décharge spontanée des électroscopes et de la différence thermique présente au-dessus des cages de Faraday et des accumulateurs d'Orgone (la chaleur latente étant l'énergie retenue dans les structures de phase ou les liens non-covalents des molécules - qui se distingue de la chaleur sensible, qu'elle soit cinétique ou électromagnétique).

Quand aux études des pulsations de l'Orgone dans le vide relatif, Reich y confondit différents régimes de décharge électrique - soit des gaz, des plasmas ou des "émissions de champ" de la cathode - qu'il observa sans pour autant les comprendre. Il identifia cependant dans les tubes VACOR des réponses de très haute fréquence lorsque ceuxci étaient excités par des bobines d'induction, réponses qu'il exploita dans le moteur à Orgone.

Durant toute cette période Reich fut souvent confronté - lorsqu'il découvrait des anomalies - à des phénomènes de transmission électriques qui semblaient "unipolaires" et avaient lieu dans des circuits ouverts. Il ne fut pas en mesure de les distinguer effectivement de l'électricité "bipolaire" opérant dans les circuits fermés ordinaires.

22 Correa PN \& Correa AN (1998, 2001) "The thermal anomaly in ORACS and the Reich-Einstein experiment: implications for blackbody theory", Akronos Publishing, Concord, Canada, ABRI monograph AS2-05.

23 Correa PN \& Correa AN $(1998,2001)$ "Comparative study of the variation in the spontaneous discharge rate of atmospheric electroscopes and electroscopes placed within 'orgone accumulators' ", Akronos Publishing, Concord, Canada, ABRI monograph AS2-06.

24 Correa PN \& Correa AN (2002) "A modified orgone accumulator (HYBORAC) as a drive for a low Delta-T Stirling engine - Part I", Infinite Energy, 41:23; also Akronos Publishing, Concord, Canada, ABRI monograph AS2-25.

25 Correa PN \& Correa AN (2002) "A modified orgone accumulator (Complete HYBORAC) as a nighttime drive for a low Delta-T Stirling engine - Part II", Infinite Energy, 42:41; also Akronos Publishing, Concord, Canada, ABRI monograph AS226.

26 Correa PN \& Correa AN (2003) "Around the clock free power from improved HYBORACs driving low delta T gamma Stirling engines - Part IV", Akronos Publishing, Concord, Canada, ABRI monograph AS2-32. 
D'après les travaux des Correas 27, ce qu'il lui manquait alors était le traitement des charges "orgonotiques" en tant que véritablement électriques, mais ambipolaires et non pas monopolaires. Précisément, les charges monopolaires sont soit négatives (électron, antiproton, etc.), soit positives (proton, positron, etc.); elles sont toujours affectées de masse et leurs courants visent à la neutralisation de la charge. Les charges ambipolaires, par contre, n'ont pas de masse et leur charge électrique oscille constamment entre des phases positives et négatives au cours de leurs déplacements longitudinaux 2829 . Les phénomènes de transmission unipolaire de l'électricité longitudinale dans les travaux de Tesla ne trouvent d'explication scientifique qu'avec la découverte des champs de charges non-inertielles ambipolaires. Il importe peu que le potentiel des charges en question soit élevé ou non, puisqu'elles ne sont pas des charges électrostatiques, lesquelles sont toujours monopolaires. En conséquence de tout ce qui précède, des charges ordinaires (monopolaires et inertielles) peuvent aussi capter une partie, ou même la totalité, de l'énergie des charges ambipolaires qui traversent leur milieu (c'est, en fait, l'effet du champ électrique). La chaleur latente n'est que le résultat d'une forme de captation de cette énergie ambipolaire, de sorte que ce qui s'accumule dans une "cage de Faraday" n'est pas de l'Orgone, comme Reich le prétendait, mais les produits de la captation de l'énergie ambipolaire (la vraie Orgone étant celle-ci) émise par le Soleil et par la profondeur de la Terre ces produits étant les chaleurs latente et sensible. Tout ceci ne nie pas le fait bien établi que la charge des électroscopes est toujours monopolaire, positive ou négative, jamais ambipolaire, en conséquence: jamais "orgonotique". Les erreurs du processus de recherche électrophysique mené par Reich ne lui ont donc pas permis l'identification physique exacte de l'énergie d'Orgone, ni même celle de son spectre

27 Correa PN \& Correa AN (2003) "Experimental Aetherometry, Vol. IIB", Akronos Publishing, University of Toronto Press, Concord, Canada.

28 Correa PN \& Correa AN (2003) "Experimental Aetherometry, Vol. IIB", Akronos Publishing, University of Toronto Press, Concord, Canada.

29 "Aetherometry", Encyclopedia Nomadica. 
de rayonnement - ce que les Correas ont fait en 1999 et publié en 200230.

Une autre grande découverte de Reich durant cette période fut le calcul de conversion des grandeurs de masse en grandeurs d'étendue, qu'il emploiera pour déterminer les résonances "orgonotiques" des masses atomiques. Il publiera ce calcul dans son dernier livre ${ }^{31}$ sous le titre La Loi Orgonomique du Pendule, mais sans écrire la formule de la conversion de la masse. En 2001, les Correas ont publié l'équation fondamentale et démontré que cette formule permet la conversion de la masse en longueur d'onde 323334 . Cette percée leur permettra de construire une nouvelle théorie de la gravitation basée sur des gravitons sans masse 3536 , un nouveau modèle toroïdal de l'électron 37 , et la découverte du spectre intégral du rayonnement cosmique comme

30 Correa PN \& Correa AN (2003) "Experimental Aetherometry, Vol. IIB", Akronos Publishing, University of Toronto Press, Concord, Canada.

31 Reich W (1957) "Contact with space", Core Pilot Press, NY.

32 Correa PN \& Correa AN $(1997,2001)$ "Non-equivalence between work performed by charge against gravity and the electric energy of the same 'charge gas'", Akronos Publishing, Concord, Canada, ABRI monograph AS2-01.

33 Correa PN \& Correa AN (2003) "The Gravitational Aether, Part I: Gravitational Orgonometry (1)", Volume II of the Aetherometric Theory of Synchronicity (ATOS), Akronos Publishing, Concord, Canada, ABRI monograph AS3-II.1.

34 Correa, P \& Correa, A (2003) "The Gravitational Aether, Part I: Gravitational Orgonometry (2)", Volume II of the Aetherometric Theory of Synchronicity (ATOS), Chapter 3, Akronos Publishing, Concord, Canada, ABRI monograph AS3II.2.

35 Correa, P \& Correa, A (2003) "The Gravitational Aether, Part II: Gravitational Aetherometry (1) - The aetherometric theory of the graviton", Volume II of the Aetherometric Theory of Synchronicity (ATOS), Chapter 4, Akronos Publishing, Concord, Canada, ABRI monograph AS3-II.3.

36 Correa PN \& Correa AN (2008) "The Gravitational Aether, Part II: Gravitational Aetherometry (9), Quantum and Subquantum Aether (Anti)Gravity; fine variation and determination of $G^{\prime \prime}$, Volume II of the Aetherometric Theory of Synchronicity (ATOS), Akronos Publishing, Concord, Canada, ABRI monograph AS3II.11.

37 Correa PN \& Correa AN (2010) "The electron mass-energy as a deformable nanometric torus", Volume III of the Aetherometric Theory of Synchronicity (ATOS), Akronos Publishing, Concord, Canada, ABRI monograph AS3-III.4. 
résultant des cascades de particules ambipolaires (les "rayons cosmiques de ultra-haute énergie") mises par la structure mouvante ("flowing lattice") de l'Éther ${ }^{38}$. Dans cette perspective, le travail de Reich fut une ébauche inachevée d'une nouvelle théorie des "gravitons oscillants" ("swing gravitons"), envisagés comme des particules d'Orgone sans masse. Reich avait d'ailleurs déclaré à Einstein qu'il avait découvert l'énergie responsable du champ gravitationnel que sa Théorie de la Relativité Générale proposait.

\section{L'Invention du "Moteur d'Orgone" et la "Clé du Cancer", 1946-1950}

\section{Retour à la table des matières}

En dépit de ses erreurs d'analyses électriques et thermiques, 1947 fut pour Reich l'année de ses plus brillantes découvertes. En effet, il existe bel et bien une autre électricité, sans masse et transmise par des radiations longitudinales, et ce même si Reich n'est pas parvenu à la comprendre ou à l'analyser comme il faudrait: il n'a identifié ni son spectre ni ses conversions. Tesla avait également échoué face aux mêmes obstacles. Mais ce que Reich connaissait alors lui suffisait pleinement pour construire un système qui couplera la méthode de transmission de l'électricité à distance de Tesla avec un nouveau circuit de captation de cette électricité à deux étages, qui la convertit pour faire tourner des moteurs spéciaux - alors tenus secrets par l'armée Américaine - nommés "Spinner Motors". Il appellera ce nouveau circuit "Facteur Y", et parfois "Fonction Y" 39. Il le gardera secret jusqu'à sa mort comme d'ailleurs tout le reste de l'appareil (la transmission Tesla, les tubes thermioniques intermédiaires, le moteur et son

38 Correa PN \& Correa AN (2008) "The Gravitational Aether, Part II: Gravitational Aetherometry (9), Quantum and Subquantum Aether (Anti)Gravity; fine variation and determination of $G^{\prime \prime}$, Volume II of the Aetherometric Theory of Synchronicity (ATOS), Akronos Publishing, Concord, Canada, ABRI monograph AS3II.11.

39 Reich, W (1949) "A motor force in orgone energy. Preliminary Communication", Orgone Energy Bull, 1:7. 
circuit de contrôle) employé dans les démonstrations qu'il fit du "Moteur à Orgone" à ceux qu'il estimait être ses plus proches collaborateurs, amis et disciples. Le "Moteur à Orgone" pouvait tourner rapidement lors des jours anticycloniques mais était erratique et inversait soudainement sa direction pendant les jours cycloniques.

En 2005, les Correas ont publié un gros livre 40 sur leurs travaux d'investigation du Moteur à Orgone, qui identifie et étudie le Facteur $Y$ en le couplant à des systèmes de transmission d'électricité ambipolaire. Ils ont obtenu deux brevets du American Patent \& Trademark Office sur leur "Aether Motor", en 2005 et 20074142 . Le Facteur $Y$ y est couplé avec le principe de transmission résonante découverte par Nikola Tesla 43 . Avec les mêmes sources d'énergie qu'avait employées Reich - y compris les tubes VACOR et l'électricité du corps humain leur méthode réplica en détail les comportements erratiques observés par Reich ainsi que les vitesses rapides et variables observés lors des jours anticycloniques. Ils ont également simplifié et perfectionné les différents circuits et composants du "moteur-convertisseur", jusqu'à entrainer la chaleur latente ambiante dans la transmission Tesla - qui contribue à produire un excès d'énergie dans la transmission - et à produire des effets moteurs constants et contrôlables. En somme, ils ont démontré que l'on peut bien obtenir de l'électricité ordinaire en captant l'électricité ambipolaire qui irradie l'espace, en particulier celle qui vient du Soleil.

Les travaux de recherche scientifique des Correas et de leurs associés ont aujourd'hui donné raison à la vision de Reich - et de Tesla qui y est conservée, critiquée et développée, même si cette vision

40 Correa PN \& Correa AN (2005) "The discovery of the Aether motor (1): the rediscovery of the Orgone Motor", Akronos Publishing, University of Toronto Press, Concord, Canada.

41 Correa PN \& Correa AN (2006) "Energy conversion systems", 30 Mai, USPTO, Pat.\#7,053,576, U.S.A.

42 Correa PN \& Correa AN (2007) "Energy conversion systems", 26 Juin, USPTO, Pat. \#7,235,945, U.S.A.

43 Tesla, N (1892) "Experiments with alternate currents of high potential and high frequency", dans "Nikola Tesla Lectures", 1956, Beograd. 
continue d'être soumise, comme hier, à la suppression à laquelle de nombreux acteurs collaborent, y compris les Reichiens actuels 44 .

C'est également lors de sa période la plus créative que Reich élaborera sa vision cosmologique - l'Orgone y étant "le vrai Ether" - dans deux nouveaux essais: L'Ether, Dieu et le Diable (1949) et La Superposition Cosmique (1950). Des phénomènes météorologiques à la création des galaxies, de la genèse des particules matérielles jusqu'aux structures des êtres vivants, Reich propose qu'il s'agisse à chaque fois d'une superposition de deux ou de plusieurs flux d'Orgone. Tout comme Einstein dans sa Théorie de la Relativité Restreinte, Reich accepte le résultat négatif de l'expérience de Michelson-Morley (1887) comme preuve qu'il n'y a pas d'Ether Luminifère stationnaire car, dit-il, l'Ether de l'Orgone est dynamique, en mouvement constant et nonélectromagnétique. Il considère aussi que l'Orgone terrestre provient d'un océan d'Orgone cosmique qui frappe constamment la terre et le système solaire, les entrainant dans un mouvement dans l'espace. Ce fut la première ébauche d'une théorie non-classique de l'Ether, mais il lui manquait la mathématique nécessaire pour y développer une analyse profonde ainsi qu'établir le lien entre tous ces processus. Reich proposa bien quelques nouvelles formules dans ce contexte - pour comprendre la formation des ouragans, des Auroras Borealis et Australis, la mutualité de la gravitation de Newton, l'analyse non-inertielle du pendulum gravitationnel, etc. - et commença même un nouveau programme de développement d'une algèbre appliqué aux problèmes scientifiques, saisis dans une perspective fonctionnaliste 454647 . Mais il ne sera pas capable d'énoncer une théorie mathématique unitaire de l'Ether

44 Askanas M (2001) "Exposé of the secret and not so-secret misery of (An)Orgonomy and Reichianism, considered in all of its aspects - spiritual, material, sexual, economic and political, and in particular scientific", Akronos Publishing, Toronto, Canada.

45 Reich W (1950) "Orgonometric equations: I. General Form", Orgone Energy Bull, 2(4):161.

46 Reich W (1951) "Orgonometric equations: II. 1. Complete Orgonometric equations", Orgone Energy Bull, 3(2):65.

47 Correa PN (2004) "Notes on MicroFunctionalism: Orgonomic, Molecular and Aetherometric Functionalism", Akronos Publishing, Concord, Canada, ABRI monograph AS1-08. 
sans masse. Il lui manquait pour cela les fonctions fondamentales de l'Orgone en tant qu'énergie ainsi que ses conversions possibles, tout autant que la découverte du spectre de l'Orgone - et à ce moment-là de son parcours, la découverte de la DOR (voir prochaine section). Sa cosmologie se réduisit alors à une grande vision inachevée et pleine d'inconsistances.

C'est aussi dans cet âge d'or, sur le front de la recherche sur les bions et la carcinogénèse, que Reich en vient à systématiser tout son travail dans un nouveau livre: La Biopathie du Cancer 48 , achevé en 1948. Il y soumet le sang de malades divers, y compris du cancer, à des tests chimiques et physiques, et compare les résultats avec ceux obtenus de donneurs sains. Il conclut à deux types de réactions: soit que la décomposition des cellules sanguines produise des bions PA (réaction B), soit qu'elle produise des bions PA plus petits et noircis, accompagnés de bacillus-T en grandes quantités (réaction $T$ ). Il isole les bacillus-T et provoque des maladies leucémoïdes de type lymphomatique sur des souris injectées avec ces préparations. Cependant - et contrairement à ce qui est souvent dit - Reich n'a jamais tenu les bacillus-T pour la cause du cancer. Il les considérait seulement comme symptômes et cofacteurs d'un processus cancéreux.

Reich fit ici des découvertes originelles sur ce qui sera plus tard (dans les années 60) connu au sujet des mycoplasmas submicroscopiques, et de leurs capacités à promouvoir des maladies leucémoïdes. Mais ce sera en dépit d'une théorie cytologique du cancer (la "théorie orgonomique") faisant appel à l'hypothèse de l'abiogénèse des eucaryotes 4950 , laquelle $n^{\prime} a$ depuis reçue aucune confirmation, bien au contraire.

Néamoins, inspiré des travaux d'Otto Warburg et d'Albert SzentGÿorgy sur le métabolisme respiratoire et l'absence de ce métabolisme

48 Reich, W (1948) "The Cancer Biopathy", Farrar, Straus \& Giroux, NY. Traduit en Fr. par Kamnitzer sous le titre "La biopathie du cancer", Payot SH, 1975.

49 Correa PN \& Correa AN (2010) "The orgonomic theory of cancer", J Biophys Hematol Oncol, 1(3):1.

50 Reich, W (1948) "The Cancer Biopathy", Farrar, Straus \& Giroux, NY. Traduit en Fr. par Kamnitzer sous le titre "La biopathie du cancer", Payot SH, 1975. 
dans la plupart des cellules cancéreuses 51 , la théorie orgonomique du cancer propose que la sympaticotonie chronique (la répression sexuelle) suffoque le corps de par l'immobilité diaphragmatique qui l'accompagne. Le premier système atteint étant le compartiment des cellules rouges, affaiblies progressivement par des seuils successifs d'asphyxie. Le manque général d'oxygène s'aggrave dans l'un ou l'autre organe où une tumeur apparaitra en premier. Reich proposa également que la leucémie soit la conséquence d'une réponse auto-immune - une réaction en quelque sorte "normale" - contre les cellules rouges affaiblies par le manque chronique d'oxygène.

Dans ces domaines de l'étiologie du cancer et de la leucémie - en y excluant la cytologie de la transformation néoplasique qu'il a proposée - les travaux de Reich pourraient bien avoir étés en avance sur son temps et le nôtre. Ce n'est que récemment que l'on a commencé à découvrir que beaucoup de leucémies produisent des anticorps contre les cellules rouges des malades, bien que la réponse à la question de savoir si cela se passe à l'origine ou comme la conséquence de la leucémie ne soit pas encore claire.

Ses approches cliniques du traitement du cancer et de la leucémie irradiation dans les accumulateurs d'Orgone 5253 , captation solaire, exercices de respiration, massage végétothérapeutique, reprise de la vie sexuelle et démontage de la cuirasse - ne seront pas suffisantes mais devront faire partie de toute guérison véritable.

51 Correa PN \& Correa AN (2010) "A unitarian biochemical and bioenergetic theory of adaptive oncogenesis: from hypoxia and energy starvation (aerobic and ambipolar) to the roles of HIF-1, IGF-I, and Vitamins C and D", J Biophys Hematol Oncol, 1(5):1.

52 Correa PN \& Correa AN (2010) "A unitarian biochemical and bioenergetic theory of adaptive oncogenesis: from hypoxia and energy starvation (aerobic and ambipolar) to the roles of HIF-1, IGF-I, and Vitamins C and D", J Biophys Hematol Oncol, 1(5):1.

53 Reich W (1939) "Bion experiments on the cancer problem", Oslo, Norvége. 


\section{La Découverte de l'énergie de la DOR (Deadly ORgone), 1950-1956}

\section{Retour à la table des matières}

Après l'âge d'or ce fut la chute, comme dans une mauvaise tragédie. Elle fut précipitée par trois facteurs. Le premier fut le retour intense de la persécution menée par les psychanalystes d'une part et les stalinistes de l'autre, auquel s'ajoutèrent les pouvoirs policiers, légaux, médicaux et médiatiques Américains. Les articles diffamatoires et mesquins de la staliniste Mildred Brady en formèrent la pierre angulaire 5455 . Brady donna libre cours aux rumeurs lancées par la psychanalyste Edith Jacobson et la Clinique Menninger; elle accusa publiquement Reich de masturber des malades dans l'accumulateur d'Orgone, et d'autres choses sordides bien pires encore. La FDA intervint en guise de justification, intervention qui en dernière analyse mènera Reich à sa mort et à sa destruction. Le second facteur fut la fragilité du support collectif du travail de Reich. Bien qu'il ait rassemblé tout un groupe autour de lui - surtout des médecins, des éducateurs et des "sponsors" (la plupart desquels ayant été analysés) - il lui manquait le soutient de scientifiques, la collaboration avec des physiciens et des biologistes. De surcroît, ceux qui le soutenaient, y inclus ses disciples, ne le comprenaient quasiment pas, se limitant à le suivre comme un chef et prêts à se dérober dès qu'ils se sentaient personnellement menacés par le Pouvoir, à cause des travaux scientifiques et les positions politiques prises par Reich. Mais le pire des facteurs de sa chute fut la recherche - classée sous la rubrique de l'Experience ORANUR - qui le conduisit tant bien que mal à la découverte de la DOR. A la suite de I'ORANUR, presque tout le monde l'abandonna, même sa femme Ilse.

54 Brady ME (1947) "The new cult of sex and anarchy", Harper's Magazine, April.

55 Brady ME (1947) "The strange case of Wilhelm Reich", The New Republic, 26 Mai. Repris dans Bulletin of the Menniger Clinic, 1948, et dans "Is the world sexually sick?", Everybody's Digest, Décembre. 
Encore une fois, on va rencontrer chez Reich - avec son concept de DOR - ce que Bergson appelait un "mixte impur", car Reich assimilera dans le concept de DOR deux réalités physiques très différentes. En quête de finances, Reich déclara aux directeurs de la Fondation Wilhelm Reich que l'irradiation d'Orgone pourrait induire une immunité énergétique contre les radiations ionisantes, et déclencher des processus physiques nouveaux capables de neutraliser la désintégration atomique ${ }^{56}$. Alors que la Guerre de Corée était sur le point de devenir un conflit nucléaire, il crut que seules ses méthodes pourraient sauver I'humanité d'une catastrophe nucléaire qu'il estimait inévitable suite à la montée du "fascisme rouge" (le stalinisme, le maoïsme). Mais quand il ramena des sources nucléaires (radium et cobalt) dans son laboratoire, où "la concentration de l'Orgone était très élevé", et les plaça dans des accumulateurs spéciaux, une réaction identique à la classique "maladie des rayons" apparut chez les personnes qui travaillaient à Orgonon, les souris du labo et même chez lui. Reich fut alors atteint d'une grave crise cardiaque (en 1951).

De retour au travail, il remarqua que la végétation autour d'Orgonon mourrait d'étranges plaies, et que des substances noires et brunes s'étaient déposées sur les pierres et rochers. Il en conclut que l'introduction de sources nucléaires dans un milieu d'Orgone concentrée provoquait une transformation de l'Orgone en son opposé, la DOR, ou "Deadly ORgone", laquelle attaque tous les vivants, les déshydrate, et attaque même le sol - la DOR devient alors pour Reich la cause de la croissance des déserts durant les derniers millénaires. Il crut également qu'il avait fait la découverte d'une nouvelle maladie du rayonnement, "la maladie ORANUR", qui serait d'après lui le résultat non pas de l'absorption de l'énergie nucléaire par les tissus, mais de la transformation de l'Orgone en DOR dans les tissus pendant "le processus de combat contre la radiation nucléaire". Il pensa même que l'induction de la maladie ORANUR en petites doses pourrait produire une immunité anti-nucléaire. Il indique que les fréquences de la DOR sont large-

56 Reich W (1951) "The Oranur Experiment, First report (1947-1951)", The Wilhelm Reich Foundation, Rangeley, ME. 
ment supérieures à celles de l'Orgone mais n'en publie nulle part les chiffres 57.

Commence alors son combat contre la production atmosphérique de la DOR qu'il estime être la source des altérations du climat ainsi que la cause des casques de "smog". Apparaît ici l'autre versant du concept (mixte-impur) de DOR: non plus comme réaction anti-nucléaire de l'Orgone, mais comme présence dans l'atmosphère de ce que l'on définit aujourd'hui en tant qu'énergie de réaction des radicaux libres 58 . Cette énergie, comme on l'a déjà dit, résulte d'une captation de l'énergie solaire ambipolaire, par la suite emmagasinée sous forme de chaleur latente dans les molécules du gaz atmosphérique. D'après les travaux des Correas, la composante DOR du spectre solaire ambipolaire a une fréquence plus haute que la composante OR (de l'Orgone) 59; c'est alors la DOR qui est responsable de la production des radicaux libres, lorsque qu'elle est absorbée par l'atmosphère 60 . Cependant, il n'y a pas de conversion directe de l'Orgone en DOR, et vice-versa. C'est plutôt leur absorption qui passe par des réactions chimiques et des émissions de photons très différentes: c'est la matière qui est convertie lorsqu'elle absorbe soit l'Orgone soit la DOR. Les Correa ont d'ailleurs établi le bilan exact de l'énergie totale du cycle atmosphéri-

57 Reich W (1951) "The Orgonomic anti-nuclear radiation project (ORANUR) report", Orgone Energy Bull, 3(4):185.

58 Correa PN \& Correa AN (2000) "Determination of the OR and DOR energies, frequencies and wavelengths driving the atmospheric allotropic cycle of oxygen, ozone and water", Akronos Publishing, Concord, Canada, ABRI monograph AS2$17 \mathrm{~B}$.

59 Correa PN \& Correa AN (1999) "The indirect 'ORgone effect' of Tesla radiation: solar spectra for ambipolar Aether and Blackbody radiation", Akronos Publishing, Concord, Canada, ABRI monograph AS2-17A.

60 Correa PN \& Correa AN (2000) "Determination of the OR and DOR energies, frequencies and wavelengths driving the atmospheric allotropic cycle of oxygen, ozone and water", Akronos Publishing, Concord, Canada, ABRI monograph AS2$17 B$. 
que allotropique de conversion d'eau-et-oxygène en ozone-et-protons, et vice-versa 61 .

Les deux composants physiques du concept de la DOR n'étaient pas conciliables. En effet, Reich avait commis une autre erreur fondamentale dans I'Experience ORANUR: d'après les Correas 62, il a oublié de prendre en compte le rôle intermédiaire des protons lors des conversions du rayonnement des rayons alpha. Rutherford lui-même avait été alerté à ce sujet quand il observa, en 1919, des changements soudains dans le comptage de particules, qui variait en fonction de la distance à la source et dépendait des matériaux interposés. Reich n'avait pas les moyens physiques pour enregistrer ces protons - ses enregistrements de particules y étaient aveugles (de même pour les neutrons). Il en suivit que le résultat clinique de I'ORANUR ne fut pas différent de l'effet connu de la maladie des rayons, et non une nouvelle forme de maladie du rayonnement. Par contre, dans la conversion atmosphérique entre substrats chimiques induite par absorption d'Orgone ou de DOR, Reich aborda un aspect nouveau de la radiation ambipolaire, et de ses fonctions dans le changement chimique de la matière.

Pour combattre la DOR atmosphérique et induire la pluie, Reich inventa le "Cloudbuster" (briseur de nuages), faisant des essais en s'attaquant aux sécheresses dans le Maine. Lorsqu'il visait le centre d'un nuage, l'engin attirait son énergie pour la conduire vers la Terre par contact avec de l'eau en mouvement. Reich prétendait agir sur un "potentiel orgonotique" des nuages, qui était alors déchargé par le biais du Cloudbuster vers le système énergétiquement plus dense de la Terre, comme si le flux orgonotique était neg-entropique. En vérité, le Cloudbuster fonctionne à la fois comme un paratonnerre pour l'électricité ordinaire des courants monopolaires, et comme un récepteur ou une antenne pour l'électricité sans masse des flux ambipolaires. Reich découvra aussi que lorsque la periphérie d'un nuage était visée, on

61 Correa PN \& Correa AN (1999, 2001) "The allotropic atmospheric cycle of oxygen, ozone and water: foundations of photo- and aetherochemistry", Akronos Publishing, Concord, Canada, ABRI monograph AS2-09.

62 Correa PN \& Correa AN (2004) "Controlled increase of the radioactivity of beta emitters could shorten their half-lives: consequences for the remediation of nuclear waste", Projet de Recherche presenté à la New Energy Foundation. 
pourrait le faire croître et provoquer la pluie. Ses recherches sur ce sujet demeurèrent plutôt subjectives; il s'y opère un décalage croissant entre les opérations du Cloudbuster et les résultats obtenus. Reich attribue au Cloudbuster des pouvoirs incroyables sans fournir aucune preuve scientifique. Il est presque impossible d'imaginer comment le fonctionnement d'un ou deux Cloudbusters pourrait altérer le flux ou la structure d'un système anti-cyclonique. C'est peut-être à cause de cette pauvreté scientifique que le Cloudbuster est devenu la plus populaire des inventions de Reich. Beaucoup de gens se réclament des effets complètement dingues obtenus de toute une sorte de Cloudbusters "perfectionnés", qui ne sont jamais identiques à ceux de Reich. C'est précisément ici que le travail de Reich sombre dans un futurisme fantastique: en dépit de sa défense de la raison scientifique, il apporta sa propre contribution à une toujours "très à la mode" tendance culturelle micro-fasciste faite de croyances irrationnelles et anti-scientifiques, qui n'a jusqu'à nos jours jamais perdu sa place centrale dans le delirium médiatique.

Tout ceci devint encore plus trouble quand Reich s'emballa avec les médias Américains au sujet des OVNI, lors des fameux "UFO flaps" des années 1950 , et se mit à croire que la cause de ses propres persécutions était due aux "CORE men" - les occupants extraterrestres des soucoupes volantes - et que ces derniers étaient la cause de la croissance constante de la DOR dans l'atmosphère et de l'expansion des déserts. Il y aurait une guerre cosmique entre les CORE men et les humains et ceux-ci n'en seraient pas conscients - seules quelques autorités le sauraient. Reich s'imagine alors que les sources nucléaires de I'ORANUR - qu'il avait enterrées à Orgonon pour neutraliser leurs effets - ne sont plus des sources de désintégration atomique, mais des sources de particules d'Orgone - ce qu'il appellera "I'effet ORUR". II réclame la découverte, en fin de compte, d'un processus physique qui neutralise la désintégration nucléaire... Les erreurs et croyances se multiplient au fur et à mesure qu'il abandonne la méthode scientifique. Reich se mit même à croire qu'il n'était pas humain - ce qui dans un sens nietzschéen sera même le cas. Il envoie des messages urgents à de nombreuses institutions de l'État Américain - au Président Eisenhower, à la AEC (Atomic Energy Commission), etc - et sent sa croyance renforcée par le fait que des pouvoirs bienveillants (le Président et 
I'USAF) accompagnent ses travaux et veillent sur lui, car la Guerre des Mondes s'approche et que seule la technologie "orgonomique" pourra nous sauver. Il place la boîte d' $O R U R$ " contenant le radium dit "neutralisé" dans le parcours du flux interne du Cloudbuster, et le transforme, selon lui, dans une arme à rayons d'Orgone, qu'il appelle le "Spacegun". Avec ce nouvel appareil, il s'embarque pour des essais de pluie dans I'Arizona (en 1955-1956) et pour le combat contre les OVNIs. D'après lui, la présence d'OVNIs s'accompagne d'émissions de DOR qui sont la cause des nuages noirs et des déserts. Le Cloudbuster ne saurait être suffisant, ne faisant qu'attirer l'énergie des nuages ou de l'atmosphère. Le Spacegun pourrait quand à lui neutraliser les champs de DOR, et en conséquence bombarder les OVNIs avec des rayons d'Orgone pour déstabiliser leur émission de DOR. Il prit des clichés qui montrent la chute d'OVNIs déguisés en de fausses étoiles, que son Spacegun aurait intercepté 63.

\section{La Période de Persécution Judiciaire Prison et Mort, 1954-1957}

\section{Retour à la table des matières}

Son isolement, la poursuite judiciaire du FDA, les attaques sournoises de ses nombreux ennemis, l'incompréhension de ses disciples et amis - leur incapacité critique et leurs trahisons fréquentes -, le silence de ses collègues scientifiques, les erreurs qu'il avait accumulées et l'incomplétude de son cuvre, tout cela contribua à la montée d'un désir paradoxal de martyre chez Reich. Il prétend même réhabiliter l'image d'un Jésus anti-nihiliste, auquel il s'identifie, et donne la crucifixion et sa propre persécution comme exemples du meurtre quotidien de la vie, en chacun de nous, en tous, et partout 64 . La percée schizophrénique s'épanouit en paranoïa, fruit des persécutions réelles. Puis la paranoïa se convertit en dépression névrotique, avec des sursis

63 Reich W (1957) "Contact with space", Core Pilot Press, NY.

64 Reich W (1953) "The murder of Christ", Farrar, Straus \& Giroux, NY; «Le meurtre du Christ», Champ Libre, Paris, 1971. 
euphoriques. Le "processus schizoïde" de Reich s'effondre quand sa santé empire et qu'il sent le poids brut de l'incompréhension tout autour de lui.

Mais rien de tout cela ne constitue une bonne raison pour brûler ses livres et écrits, pour l'emprisonner cruellement, sinon l'assassiner! Ce qu'il n'y a certainement pas dans tout ce processus, c'est de doute envers l'existence d'une vraie conspiration menant la FDA (Food and Drug Administration), I'APA (American Psychiatric Association), I'AMA (American Medical Association), les tribunaux de Maine et bien d'autres gens et institutions (comme la Clinique de Karl Menninger ${ }^{65}$ ) engagée dans le but fasciste de détruire et le travail et la personne de Reich. Pour s'en convaincre, Il suffit de voir comment Peter Mills l'avocat de Reich - devint au cours du procès le juge qui le condamna! Il est remarquable que le mot d'ordre contre Reich ait été donné par le Dr. Ewen Cameron 66 67, alors Président de l'APA, conseiller de Dulles et "scientifique" de la CIA, lequel menait à la même période des expériences parfaitement nazies, effectuées sans la permission des "malades", recourant au LSD et à d'autres drogues sur toutes sortes de gens dans le but de prouver sa thèse ridicule du "Candidat Manchourien".

Le procès intenté par la FDA (Food and Drug Administration) en 1954 se concentra sur (1) le transport d'appareils de traitement - les accumulateurs d'Orgone à usage médical - à travers les États Américains sans licence et sans avoir démontré leur effet ou capacité thérapeutique, et sur (2) la pratique de cures médicales sans un permis médical (bien que médecin, Reich n'était pas licencié et reconnu comme tel aux États-Unis). On l'accusa de double fraude, commerciale et médicale. Il ignorera ces injonctions et sera arrêté deux fois. Ayant perdu le procès sur la première injonction, Reich refusera de reconnaître la légitimité du tribunal. De plus - et à ce propos il a plutôt raison - il

65 Boadella D (1973) "Wilhelm Reich - the evolution of his work", Arkana, Londres, p. $268 \mathrm{ff}$.

66 Sharaf M (1983) "Fury on earth - a biography of Wilhelm Reich", St. Martin's Press, NY, p. 277.

67 Boadella D (1973) "Wilhelm Reich - the evolution of his work", Arkana, Londres, p. $268 \mathrm{ff}$. 
refuse de reconnaître la compétence d'un tribunal quel qu'il soit (pas seulement le tribunal du Judge Mills) pour juger ou évaluer des découvertes scientifiques, et de surcroît les siennes en particulier. La vérité, qu'elle soit dite, c'est que le FDA na jamais étudié ou examiné le fonctionnement des accumulateurs d'Orgone. Le FDA commissionna une seule étude 68 qui ne réussit pas à reproduire les conditions nécessaires ni pour observer, ni pour mesurer l'anomalie thermique. Dans l'ambiance d'alors - chasse aux sorcières du maccarthysme et psychiatrisation de la pensée -, ce ne fut pas difficile de détruire Reich. En 1956, il sera condamné pour outrage à la cour à deux années d'emprisonnement. On l'envoie à Lewisburg, pénitencier fédéral où, d'après ses lettres, il travaille à un nouveau livre intitulé Création, lequel serait le sommet des ses équations orgonomiques. Il meurt cependant en Mai 1957 dans des conditions mystérieuses, officiellement d'une occlusion coronarienne, mais d'après le seul co-détenu qu'il ait eu à Lewisburg, à la suite d'un empoisonnement 69 . Son dernier manuscrit Création, aurait officiellement été brûlé par l'autorité du pénitencier. S'effondra alors pour toujours ce grand lutteur antifasciste, et son dernier travail sombra avec lui dans les flammes du nouveau fascisme Américain. Jusqu'à nos jours, seul le dossier du FBI sur le cas Wilhelm Reich a été ouvert au public 70 , et il ne contient rien de nouveau à propos des machinations responsables de la poursuite impitoyable de la personne de Reich et de son travail.

En 1944, Reich écrivit ce commentaire sur son cuvre dans son journal, se sentant déjà isolé du monde humain: "Une perçée tardive: la relation avec mon travail est la seule que ne $m$ 'ait pas jamais trahi. Avec amour elle me renvoya tout ce qu'avec amour je lui ai donné" 71 .

68 Smythe A (1949) "Orgone accumulator", J Am Med Association, 8 Janvier.

69 Dadoun R (1975) "Cent fleurs pour Wilhelm Reich", Payot, Paris, pp. 32-33.

70 FOIA USA, "FBI file on Dr. Wilhelm Reich", Bufile:100-14601, Section 1.

71 Note du 30 Séptembre, 1944, dans "American Odyssey - letters and journals, 1940-1947", Farrar, Straus \& Giroux, NY, 1999, p. 249. 


\section{L'Influence des Travaux de Reich, 1957-2011}

\section{Retour à la table des matières}

On peut toujours trouver des suiveurs de Reich, dans ce domaine-ci ou celui-là... L'évolution générale du concept d'Orgone fut sa dissolution effective comme concept scientifique - jusqu'à très récemment. L'Orgone devint ainsi une métaphore d'autres métaphores, soit celle de Dieu, de la force du vivant, ou de la Libido. Pire encore, elle se dissoudra dans une pluralité d'acceptions mystiques: du Ch'i au Prana. Son effet métaphorique dépendait en fait de son abandon par le discours scientifique - ce qui fut précisément le cas. Le nom "Orgone" a ainsi acquis de nos jours des sens contradictoires et pseudoscientifiques, qui ignorent tous les sens précis - y compris ceux qui en fin de compte se réfèrent à des erreurs - que Reich lui avait donné.

Peu est resté de l'héritage intellectuel et scientifique de Reich. Il n'aura eu aucun suiveur "béni". En dépit des tendances pro-Soviétiques d'Alexander Neill - I'éducateur d'enfants qui fonda le Summerhill College - ses nouvelles méthodes de formation furent fortement influencées par Reich. L'amie de Reich, la psychanalyste Norwègienne Nik Hoel, a aussi continué à employer des techniques analytiques découvertes par Reich, qui influencèrent aussi Mélanie Klein.

Après la mort de Reich, il y eut de nombreuses luttes entre diverses factions, chacune se réclamant d'une orthodoxie reichienne. Sa fille, Eva Reich, avec laquelle Reich se disputait fréquemment, ne voulut devenir l'exécutrice de son testament. Cette fonction fut prise par Mary Higgins, compagne riche de Chester Raphael, un des médecins analysés et formés par Reich, lequel domina alors le destin du patrimoine de Reich. Les groupes formés autour d'Eva, et autour d'Elsworth Baker (un autre médecin analysé et formé par Reich, qui écrira sa pauvre version de l'analyse caractérielle ${ }^{72}$ ) - dans I'American College of Orgonomy que publia le regrettable Journal of Orgonomy - se

72 Baker E (1967) "Man in the trap", Avon Books, NY. 
disputèrent "la légitimité reichienne" avec le group de Higgins et Raphael pendant des décennies. En plus, d'autres personnes de l'entourage de Charles Kelley répandaient leurs visions simplistes de l'Orgone. Rien de substantiel ne sortit de ces factions - aucune recherche scientifique ni aucune confirmation réelle des découvertes de Reich, ni quelque critique constructive sur quoi que ce soit. Au contraire, ce qui fut alors publié de nouveau sur Reich dégradait ses travaux en les teintant d'un subjectivisme hallucinatoire, comme un présage du mouvement New-Age.

Les biographies de Reich ont été pour la plupart également médiocres. Quelques-unes sont vraiment pitres 73 , d'autres souffrent d'une proximité œdipianisante insidieuse (come celle de Sharaff ${ }^{74}$ ), d'autres encore sont teintes d'une religiosité suspecte (comme celles de Mann 75 ou de Raknes ${ }^{76}$ ). Sur toutes les questions scientifiques elles sont totalement mystificatrices. Ce que Peter Reich, son fils psychanalysé dès l'enfance, a écrit au sujet de Reich dans son $A$ la recherche de mon père: rêves éclatés est peut-être un des pires résultats de la pure imagination œedipienne se donnant libre cours. L'exception fut le livre écrit par David Boadella 77 , aussi éducateur - dans I'Abbotsbury School en Dorset - et l'éditeur du journal Energy and Character. Il y tente de faire justice à la science de Reich, mais n'étant pas scientifique, il ne peut pas la comprendre en profondeur, et encore moins en faire une évaluation critique. Il se réduit alors à l'apologie de Reich.

Beaucoup de thérapeutes se sont également réclamés des pratiques reichiennes de la végétothérapie, de l'analyse caractérielle, ou de la thérapie d'Orgone - comme la "Bioenergetics" de Alexandre Lowen ou de John Pierrakos, la Somatothérapie de Richard Meyer (Strasbourg), ou l'Orgonthérapie de Gérard Guasch (Paris). On peut pas faire ici le

73 Wilson, C (1981) "The quest for Wilhelm Reich", Granada, Angleterre.

74 Sharaf M (1983) "Fury on earth - a biography of Wilhelm Reich", St. Martin's Press, NY, p. 277.

75 Mann WE (1973) "Orgone, Reich and Eros", Simon \& Schuster, NY.

76 Raknes O (1970) "Wilhelm Reich and Orgonomy", Penguin Books, NY.

77 Boadella D (1973) "Wilhelm Reich - the evolution of his work", Arkana, Londres, p. $268 \mathrm{ff}$. 
bilan de ces thérapies, quoiqu'aucune d'entre elles ne poussa plus loin la pratique reichienne d'une clinique psychosomatique.

Dans les arts, l'influence de Reich fut très mince et aussi médiocre. Kate Bush fut une grande artiste, mais sa chanson Cloudbusting (1985) est plutôt minable, seulement comparable à la superficialité dérisoire du film de Dusan Makavejev WR Mysteries of the Organism (1971), ou aux caricatures simplistes que Robert Anton Wilson fait dans sa pièce Wilhelm Reich in Hell (1987). La série des "Aktion" (1963-1973) de l'artiste viennois Otto Muehl se réfère régulièrement à Reich. Des titres tels que Fest des psycho-physischen Naturalismus ou Psycho-Motorische Geräuschaktion sont à ce propos sans équivoque. Müehl pourrait être défini comme un "artiste Reichien": il a utilisé comme fer de lance contre la société viennoise une partie des théories de La Révolution Sexuelle ainsi que d'autres idées de Reich, en les reprenant à son compte - et ce, tout en partageant avec les Reichiens une grande mécompréhension de son œuvre.

Reich influença le mouvement de l'antipsychiatrie, notamment Ronald Laing, et David Cooper qui a même écrit une critique de la fonction centralisatrice de l'orgasme dans la théorie de la sexualité de Reich. Reich influença aussi Roger Gentis et la psychiatrie de secteur tout comme il eut une influence profonde dans la création de la "schizoanalyse" de Deleuze et Guattari 78 , qui prit le relai de l'antipsychiatrie à l'époque des années 1970-1985.

D'autre part, la relation proposé par Reich entre l'économie libidinale comme superstructure et l'économie politique comme infrastructure sera reprise avec rigueur par Guy Debord dans son livre La Société du Spectacle 79 , et dans sa conception freudomarxiste d'une superstructure autonomisée ("la matérialisation de l'idéologie"). Paradoxalement - en vue de ses conceptions hégéliennes et ouvertement fascisantes - la même conception est aussi proposée en détail dans le bouquin Reich - Mode d'Emploi de Jean Pierre Voyer.

78 Deleuze G \& Guattari F (1973) "L'anti-Oedipe - capitalisme et schizophrénie", Les Éditions de Minuit, Paris.

79 Debord G (1967) "La société du spectacle", Paris. 
On peut dire que l'influence de Reich sur Deleuze et Guattari fut tout autre que freudomarxiste, car à la différence des exemples qui précèdent, leur analyse sociologique et psychiatrique des sociétés humaines 80 rejette le schéma freudomarxiste. L'influence de Reich en France est probablement en grande partie le résultat des efforts de dissémination de Roger Dadoun, qui écrivit l'un des meilleurs livres sur Reich ${ }^{81}$. Mais en dépit de ses bonnes intentions, Dadoun manque souvent de sens critique et de capacité à apprécier les faits en ce qui concerne les découvertes scientifiques et les erreurs de Reich. En outre, le marxisme diffus de Dadoun (un certain maoïsme) se manifeste un peu partout dans son livre.

Michel Foucault 82 a fait une caricature illégitime de la sexologie reichienne, en grande partie en raison du rejet manifeste de l'homosexualité fait par Reich à cause de son idéal génital. A l'extrême opposé de cette réaction se trouve James DeMeo, géographe Américain qui, en dépit de toutes les découvertes de l'ethnographie moderne, essaya de prouver l'existence du Matriarcat et proposa une thèse selon laquelle une guerre menée par les fondateurs pervers et homosexuels du Patriarcat est responsable de la désertification du Sahara ${ }^{83}$. Selon lui, il n'y a de prostitution ou d'homosexualité que dans le Patriarcat.

Toutes les influences "révolutionnaires" de Reich sont aujourd'hui bien et bel révolues. Le Reich New-Age est devenu l'archétype d'une icône "à tout faire". Un tas de choses imaginaires ont été écrites au sujet de Reich, la plupart relevant d'un profond manque d'intelligence ("grasp"). Des types prétendent par exemple que le Facteur Y c'est "toi" ("Yol") pour vendre de la potion magique à ajouter au carburant, d'autres disent qu'ils obtiennent les bions SAPA par leur seul pouvoir de prévisualisation. D'autres encore pédalent dans des conversations d'outretombe avec un Reich revenant! Un cirque chaotique de petits

80 Deleuze G \& Guattari F (1973) "L'anti-Oedipe - capitalisme et schizophrénie", Les Éditions de Minuit, Paris.

81 Dadoun R (1975) "Cent fleurs pour Wilhelm Reich", Payot, Paris, pp. 32-33.

82 Foucault M (1976) "La volonté de savoir", Éditions Gallimard, Paris.

83 Demeo, J (1998) "Saharasia", OBRL Inc, Greensprings, Oregon. 
goupuscules, de cliniques alternatives, de petites modes et de petits meneurs d'hommes...

Il se peut qu'il y ait encore des gens ou des groupes qui fassent des études sérieuses sur Reich, comme le Cercle d'Études Wilhelm Reich qu'anime Jacques Lesage de La Haye. Mais, dans tout ceci il n'y a aucun travail scientifique sur l'Orgone, aucune vérification systématique des preuves expérimentales - physiques, chimiques, biophysiques et biochimiques - de son existence, et encore moins des articulations scientifiques et cliniques de ses fonctions. On peut dire que les Correas remplirent ce rôle dès 1996 84, mais dans leurs travaux sur l'Étherométrie, ils ne se définissent ni comme des reichiens, ni en tant que suiveurs de Reich - que ce soit à cause du fait que leur travail relève de bien d'autres influences scientifiques et qu'il adopte d'autres lignes de recherche; ou du fait qu'ils aient marqué de nombreuses différences critiques envers les concepts et fonctions de l'orgonomie: c'est que leur vérification systématique a confirmé ou infirmé leur validité. C'est encore de par le fait que les avancées scientifiques et technologiques que les Correas - ainsi que la science actuelle - ont effectué, se situent au-delà de celles de Reich 85 . Ils ont cependant traité Reich avec le respect qu'il mérite et dans l'esprit qu'il a toujours voulu induire: un esprit scientifique ouvert; ils ont démontré que cet homme passionné et passionnant sera un jour reconnu pour tous ses efforts - même ceux qui étaient des erreurs - et surtout pour la découverte initiale que lui et Tesla ont faite d'un Éther sans masse et ambipolaire.

\section{Fin du texte}

84 Correa PN \& Correa AN (1996) "The Correa/Reich Affair - Anatomy of the Sensationalist Misrepresentations Regarding the Work of the Correas and That of Dr. Wilhelm Reich".

85 Correa PN \& Correa AN (2006) "50 years after the death of Wilhelm Reich", J Aetherom Res, 1(7):1. 